OPEN ACCESS

Edited by:

Jianfeng Mao,

University of Wollongong, Australia

Reviewed by:

Hao Liu,

University of Technology Sydney,

Australia

Xiayin Yao,

Ningbo Institute of Materials Technology \& Engineering, CAS,

China

Wei Li,

Fudan University, China

*Correspondence:

Kai Liang

Kliang@dhu.edu.cn

Wei LuO

wluo@dhu.edu.cn

Jianping Yang

jianpingyang@dhu.edu.cn

Specialty section:

This article was submitted to Electrochemical Energy Conversion and Storage,

a section of the journal

Frontiers in Energy Research

Received: 28 April 2020

Accepted: 09 June 2020

Published: 21 July 2020

Citation:

Qian X, Zhang F, Zhao Y, Liang K, Luo W and Yang J (2020)

Polydopamine-Derived Carbon: What a Critical Role for Lithium Storage?

Front. Energy Res. 8:140.

doi: 10.3389/fenrg.2020.00140

\section{Polydopamine-Derived Carbon: What a Critical Role for Lithium Storage?}

\author{
Xiaoyong Qian', Fangzhou Zhang ${ }^{2}$, Yuye Zhao ${ }^{2}$, Kai Liang ${ }^{1 *}$, Wei Luo ${ }^{2 *}$ and \\ Jianping Yang ${ }^{2 *}$
}

\begin{abstract}
1 Key Laboratory of Science and Technology of Eco-Textiles, Ministry of Education, College of Chemistry, Chemical Engineering and Biotechnology, Donghua University, Shanghai, China, ${ }^{2}$ State Key Laboratory for Modification of Chemical Fibers and Polymer Materials, College of Materials Science and Engineering, Donghua University, Shanghai, China
\end{abstract}

Polydopamine-derived carbon materials have attracted tremendous attention owing to nitrogen heteroatom doping. However, it is challenging to estimate the effect of the morphology and porosity of the polydopamine-derived carbon for lithium storage. Here, we designed three polydopamine-derived carbon materials with different morphologies and porosity: carbon nanospheres (CNs), mesoporous carbon nanospheres (MCNs), and bowl-like mesoporous carbon nanoparticles (BMCNs) to evaluate the electrochemical performance. Such a bowl-like mesoporous structure combines multiple advantageous features, including mesoporous channels and shorter transmission distance, thus delivering a high specific capacity. In addition, our work provides new insight into the electrochemical performance of polydopamine-derived carbon and a useful reference for its future application in high-performance lithium ion battery (LIB) electrode materials.

Keywords: polydopamine, porous carbon, mesoporous structure, morphology, lithium storage

\section{INTRODUCTION}

With increasing aggravation of energy crisis and environment pollution, the development of an environmentally friendly new energy to replace traditional fossil energy is essential for building a sustainable energy structure and a friendly eco-environment. Lithium ion batteries (LIBs) are the most competitive candidates of energy storage systems (ESS) and have a wide application in portable electronic devices and electric vehicles (Tarascon and Armand, 2001; Jiang et al., 2015; Liu et al., 2015; Li et al., 2019; Zhu et al., 2019). However, graphitic carbon, as currently the standard commercial anode material for LIBs, possesses low theoretical capacity ( $372 \mathrm{~mA} \mathrm{~h} \mathrm{~g}^{-1}$ ) and undesirable rate performance, which limit its application in a high-energy system (Dai et al., 2019; Zheng et al., 2019; Rao et al., 2020). Nitrogen-doped carbon nanomaterials have been widely explored as alternatives to commercial graphitic materials, showing high reversible capacity, high-rate performance, and high cyclic capability. A variety of nitrogen-doped carbon materials have been obtained by using urea (Chen et al., 2018; Sun et al., 2019), polypyrrole (Qie et al., 2012; Liu et al., 2016), polydopamine (Jian et al., 2019; Qian et al., 2020), and biomass sources (Lang et al., 2019; Shen et al., 2019; Zhao et al., 2019) as precursors. Among these materials, polydopamine-derived carbon materials have been widely applied in the anode/cathode field due to that it can easily coat on virtually any surface by self-polymerization of dopamine in aqueous media. For instance, the Si@void@C “yolk-shell” structure was fabricated via a sacrificial template process, which delivered a high capacity of $\sim 1500 \mathrm{~mA} \mathrm{~h} \mathrm{~g}^{-1}$ at a current density of $1 \mathrm{~A} \mathrm{~g}^{-1}$ 
(Liu et al., 2012). Wang and coworkers designed interconnected $\mathrm{N} / \mathrm{O}$ co-doped porous rod-like nanocapsules (N/O-CNCs) through a sacrificial template process, and a capacity of $310 \mathrm{~mA} \mathrm{~h} \mathrm{~g}^{-1}$ was obtained at $30 \mathrm{~A} \mathrm{~g}^{-1}$ after 11000 cycles (Wang L. et al., 2019). Recently, Mai and coworkers reported sandwichstructured mesoporous polydopamine (mPDA)/reduced graphene oxide ( $\mathrm{rGO}$ ) nanosheets and found that this composite electrode could display a high specific capacity (Wang $\mathrm{N}$. et al., 2019). In addition, compared with the traditional hollow structure, the bowl-like morphology has received extensive attention due to its unique structural advantages (Liang et al., 2015; Qian et al., 2020). However, to the best of our knowledge, the influence of the morphology and porosity of the polydopamine-derived carbon for lithium storage have not been thoroughly evaluated as anode materials.

Herein, we have designed three polydopamine-derived carbon materials with different morphologies and porosities: carbon nanospheres (CNs), mesoporous carbon nanospheres (MCNs), and bowl-like mesoporous carbon nanoparticles (BMCNs). Importantly, the effect of the morphology and porosity of the polydopamine-derived carbon for lithium storage were evaluated in detail. We find that the mesoporous structure can shorten the diffusion path of $\mathrm{Li}$ ions and electrons in the electrode and accelerate the diffusion rate of the electrolyte. In addition, the bowl-like morphology has a shorter transmission distance than nanospheres, which can shorten the lithium ion and electron transmission paths. Therefore, BMCNs combine the advantages of both mesoporous channels and shorter transmission distance so that it can effectively relieve the stress contributing to superior long-term cycling stability. The obtained BMCN electrode delivers a large reversible capacity of $300 \mathrm{~mA} \mathrm{~h} \mathrm{~g}^{-1}$ after 1000 cycles, revealing outstanding long-term cycle stability at $2 \mathrm{C}$.

\section{RESULTS AND DISCUSSION}

The overall fabrication process for CNs, MCNs, and BMCNs is schematically illustrated in Figure 1a. First, CNs were synthesized by the polymerization of dopamine hydrochloride (DA) under the condition of basic aerobic conditions using ammonia as a catalyst. Second, the low-block copolymer F127 is used as the template, DA as the carbon source. TMB acts as the medium in the ethanol/water system, which interacts with the hydrophobic PPO segment of F127 and DA molecules, respectively. This promotes the formation of F127/TMB/DA composite micelles. Continuous cooperative assembly promotes the formation of the initially formed composite micelles along the particle interior to form mesopores. The polymerization reaction under oxygen conditions continues to grow, and finally MCNs were formed by carbonization treatment in an $\mathrm{N}_{2}$ atmosphere. Third, TMB was ultrasonically transformed into an emulsion to form an "oilin-water" system. A block copolymer F127/TMB/DA composite micelle is formed at the $\mathrm{TMB} /$ water interface; this seed-mediated anisotropic growth process yields BMCNs. The color changes of the synthesis process are shown in Supplementary Figure S1.

The SEM image (Figure 1b) and TEM images (Figure 1c and Supplementary Figure S2) show that CNs have particle sizes between 200 and $500 \mathrm{~nm}$ and cross-linked with each other; the surface is smooth, and no mesoporous channels are observed. MCNs obtained are very uniform with an average size of about $200 \mathrm{~nm}$, and the ultrasmall mesopores are distributed on the sphere surface (Figures 1d,e and Supplementary Figure S3). The SEM image (Figure 1f) and TEM images (Figure $\mathbf{1 g}$ and Supplementary Figure S4) show that BMCNs clearly display a bowl-like structure with an even particle diameter of $\sim 250 \mathrm{~nm}$, and uniform mesopores can be distinctly observed.

$\mathrm{X}$-ray diffraction (XRD) patterns (Supplementary Figure S5) of the three samples suggest that two broad peaks at about $23.5^{\circ}$ and $43.7^{\circ}$ were assigned to partly graphitized (002) and quasi-amorphous (100) structure diffraction modes (Jian et al., 2019; Nan et al., 2019). The Raman spectra of CNs, MCNs, and BMCNs are shown in Supplementary Figure S6. It is known that the two characteristic peaks located at $\sim 1350$ and $\sim 1590 \mathrm{~cm}^{-1}$ correspond to disordered $\mathrm{sp}^{3}$ carbon (D band) and graphitic $\mathrm{sp}^{2}$ carbon (G band), respectively (Gayathri et al., 2019; Hu et al., 2019). In addition, the intensity ratios of $D$ and $G$ bands $\left(I_{D} / I_{G}\right)$ for CNs, MCNs, and BMCNs are 1.03, 1.04, and 1.05, respectively, indicating a relatively superior graphitic degree.

The chemical composition and electronic structure of the three samples were analyzed by X-ray photoelectron spectroscopy (XPS). Figure 2A presents three typical peaks of $\mathrm{C} 1 \mathrm{~s}, \mathrm{~N} 1 \mathrm{~s}$, and O1s without any impurities in three samples. The spectrum of $\mathrm{C} 1 \mathrm{~s}$ exhibits that the primary characteristic peaks at 284.6, 285.9, and $289.1 \mathrm{eV}$ denote the $\mathrm{C}-\mathrm{C}, \mathrm{C}-\mathrm{N}$, and $\mathrm{O}-\mathrm{C}=\mathrm{O}$ of $\mathrm{CNs}, \mathrm{MCNs}$, and $\mathrm{BMCNs}$ (Figure 2B), respectively (Zheng et al., 2014; Chu et al., 2018; Shi et al., 2020). Four obvious peaks of $\mathrm{N}$ 1s at 398.4, 399.7, 400.6, and $402.4 \mathrm{eV}$ are related to pyridinic $\mathrm{N}$, pyrrolic $\mathrm{N}$, graphitic $\mathrm{N}$, and oxidized $\mathrm{N}$ in Figure 2C (Chen et al., 2016; Liu et al., 2017; Peng et al., 2019). The doping of nitrogen is conducive to enhancement of the electrochemical activity and conductivity of the material (Ai et al., 2013; Mao et al., 2018; Wang et al., 2018). Moreover, the atomic ratio in Figure 2D presents that the proportions of the three elements $\mathrm{C}, \mathrm{N}$, and $\mathrm{O}$ of $\mathrm{CNs}, \mathrm{MCNs}$, and BMCNs are basically the same. The above results indicate that the phase composition of CNs, MCNs, and BMCNs is identical, and there are only differences in pore size and morphology. The specific surface area and porosity distribution of MCNs and BMCNs were obtained from the $\mathrm{N}_{2}$ adsorption/desorption analysis. Figure 2E shows that both MCNs and BMCNs display representative typeIV isotherms with an evident hysteresis loop, indicating the presence of mesoporous structures (Hong et al., 2019; Tian et al., 2019; Yin et al., 2019). The Brunauer-Emmett-Teller specific surface area of BMCNs was $44.2 \mathrm{~m}^{2} \mathrm{~g}^{-1}$, which was larger than that of MCNs $\left(19.9 \mathrm{~m}^{2} \mathrm{~g}^{-1}\right)$. As shown in Figure 2F, the pore size distribution curves calculated using the Barrett-Joyner-Halenda model confirmed that the pore diameters of BMCNs are mainly distributed around $3.1 \mathrm{~nm}$, which are larger than MCNs $(2.2 \mathrm{~nm})$. The results show that BMCNs have richer mesoporous channels, which will facilitate the contact between the active material and the electrolyte and accelerate $\mathrm{Li}^{+}$and electron transport.

We investigated and compared the electrochemical properties of CNs, MCNs, and BMCNs as candidate anode materials for LIBs. The Li-ion reaction behavior of the three electrodes was 

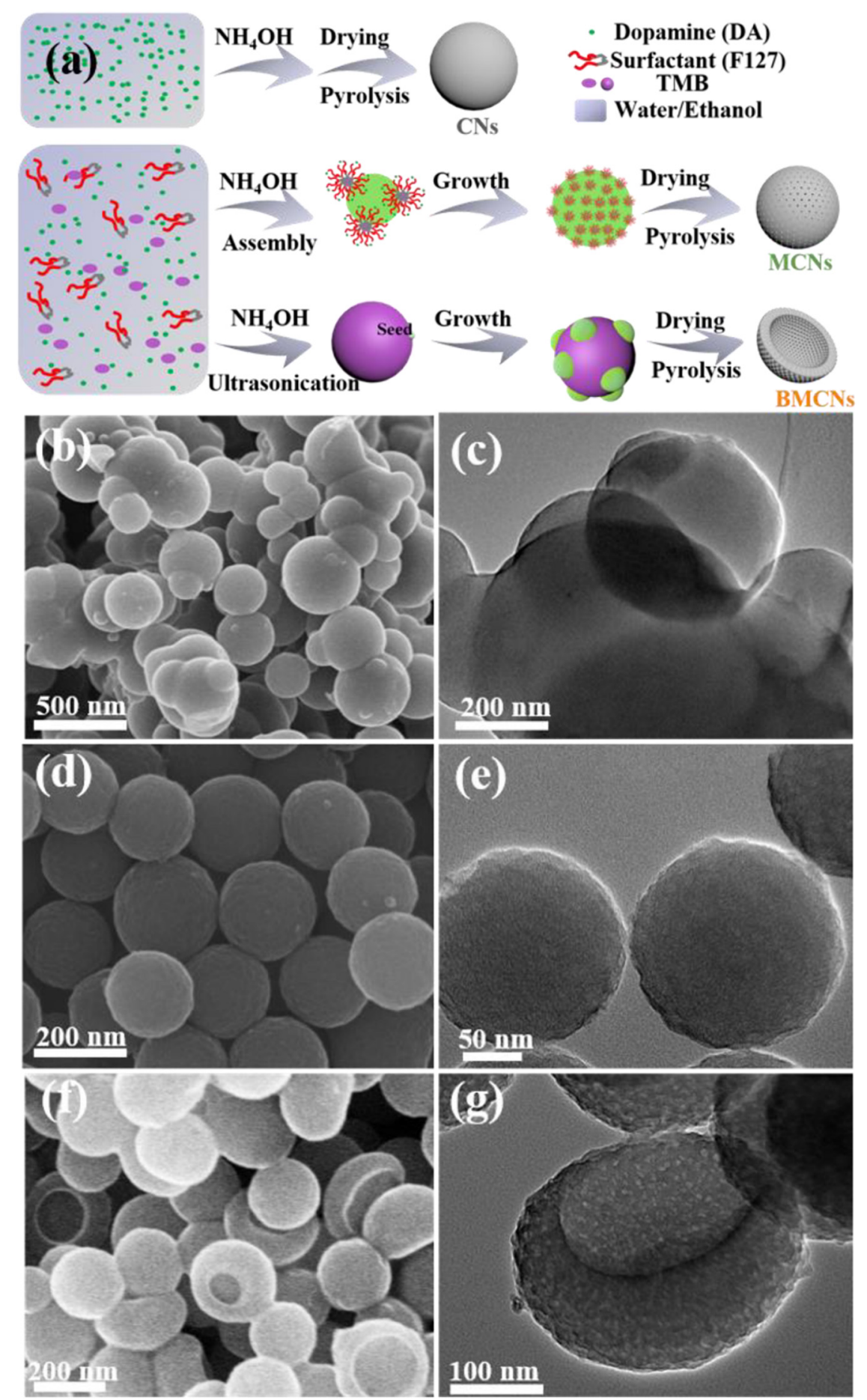

FIGURE 1 | (a) Schematic illustration for the fabrication procedure of CNs, MCNs, and BMCNs. FESEM and TEM images of (b-c) CNs, (d-e) MCNs, and (f-g) BMCNs.

tested via cyclic voltammograms (CVs), as exhibited in Figure 3A and Supplementary Figure S7. The 0.9-V peaks can correspond to the decomposition of the electrolyte followed by the formation of a solid electrolyte interface (SEI) layer (Cui et al., 2019; Xu et al., 2019; Boyjoo et al., 2020). The 0.01-V cathodic peak is ascribed to the intercalation and deintercalation of $\mathrm{Li}$ ions into graphene-like planes (Liu et al., 2018; Ould Ely et al., 2019; Rao et al., 2020). The galvanostatic discharge/charge curves of the BMCNs at $0.1 \mathrm{~A} \mathrm{~g}^{-1}$ are presented in Figure 3B. The BMCN electrode delivers high first-cycle discharge and charge capacities 
A

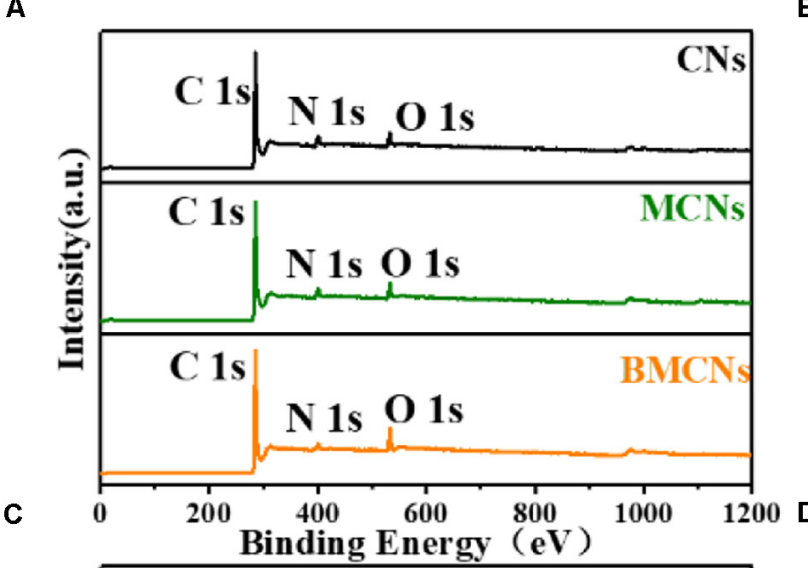

B
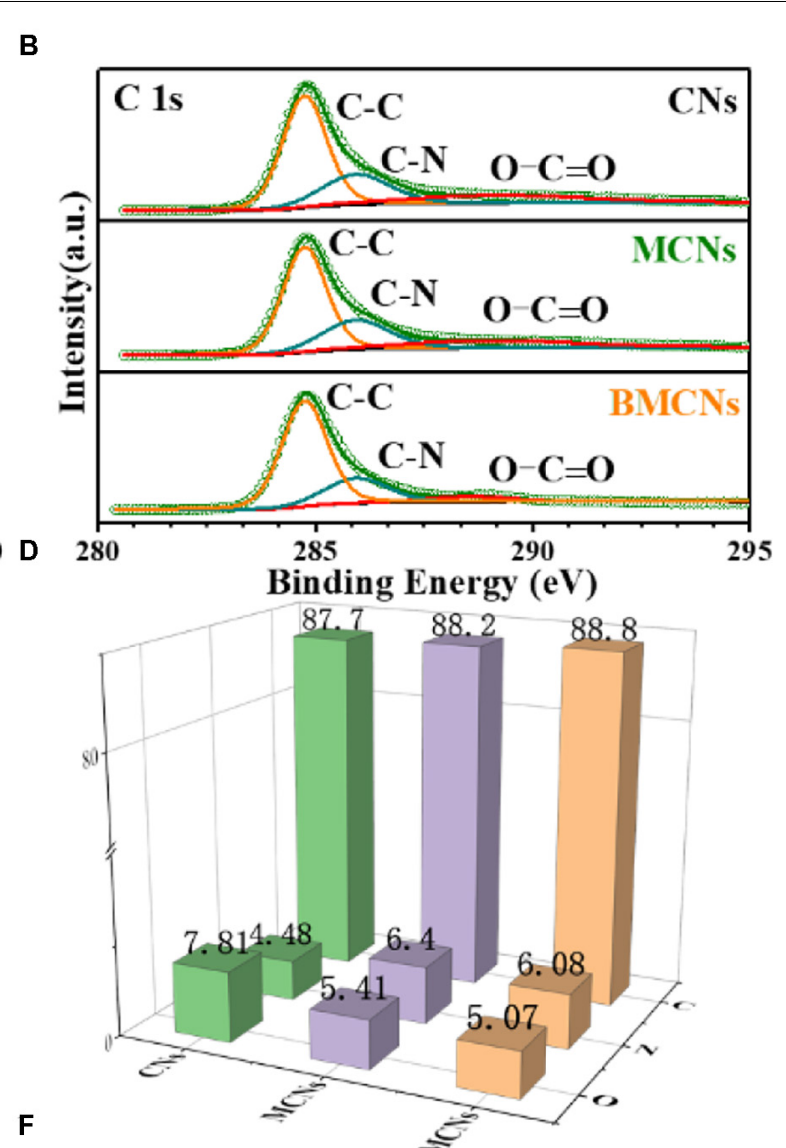

$F$

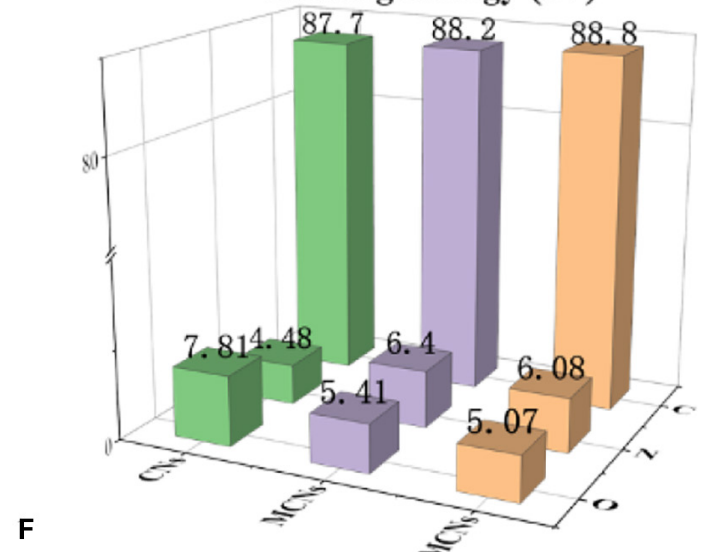

E

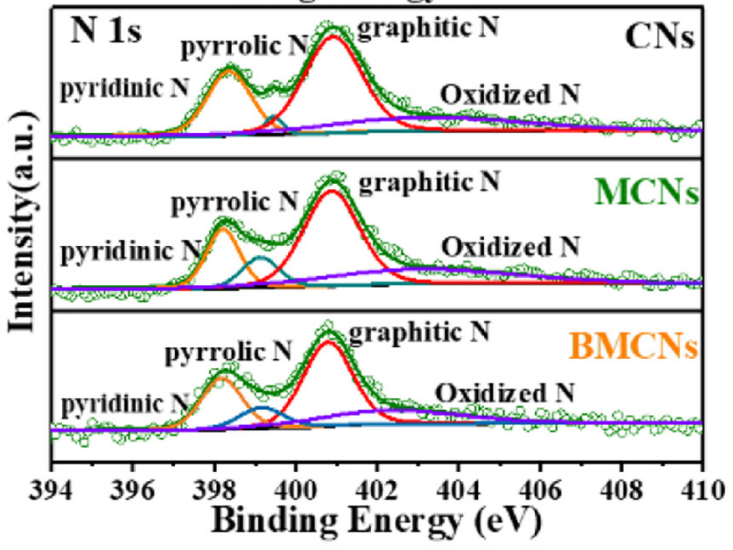

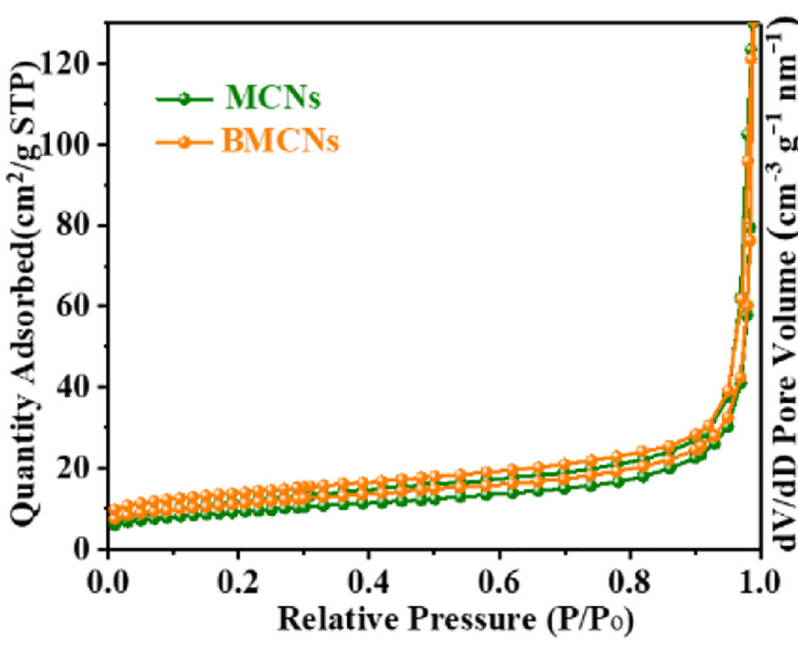

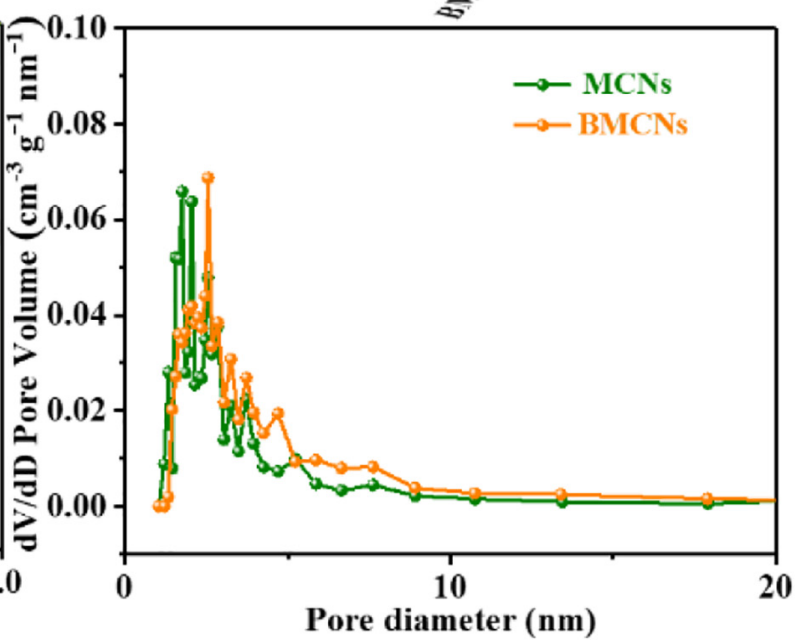

FIGURE 2 |XPS spectra for CNs, MCNs, and BMCNs: (A) Survey spectrum and high-resolution spectra of (B) C 1s, (C) N 1s, and (D) atomic ratio. (E) N2 adsorption/desorption isotherms of MCNs and BMCNs, (F) pore size distribution.

of 868 and $507 \mathrm{~mA} \mathrm{~h}^{-1}$, respectively, suggesting an initial Coulombic efficiency (ICE) of $58.4 \%$. The initial Coulombic efficiencies of $\mathrm{CN}$ and $\mathrm{MCN}$ samples are $61.4 \%$ and $55.3 \%$, respectively. The irreversible capacity loss may be originated from the formation of an SEI layer during the first cycle and electrolyte decomposition (Zhang et al., 2018; Zou et al., 2018; Liu et al., 2020). Figure 3C shows the cycling performance of CNs,
MCNs, and BMCNs at $0.1 \mathrm{~A} \mathrm{~g}^{-1}$. After 100 cycles, the BMCN electrode delivers a significant reversible capacity up to $371 \mathrm{~mA} \mathrm{~h}$ $\mathrm{g}^{-1}$. In stark contrast, $\mathrm{CN}$ and $\mathrm{MCN}$ electrodes can only deliver 277 and $171 \mathrm{~mA} \mathrm{~h} \mathrm{~g}^{-1}$ after 100 cycles, respectively. Discharge capacity and Coulombic efficiency of the first five cycles of $\mathrm{CN}$, $\mathrm{MCN}$, and BMCN electrodes are presented in Figure 3D. The $\mathrm{BMCN}$ electrode performs the best and delivers high discharge 

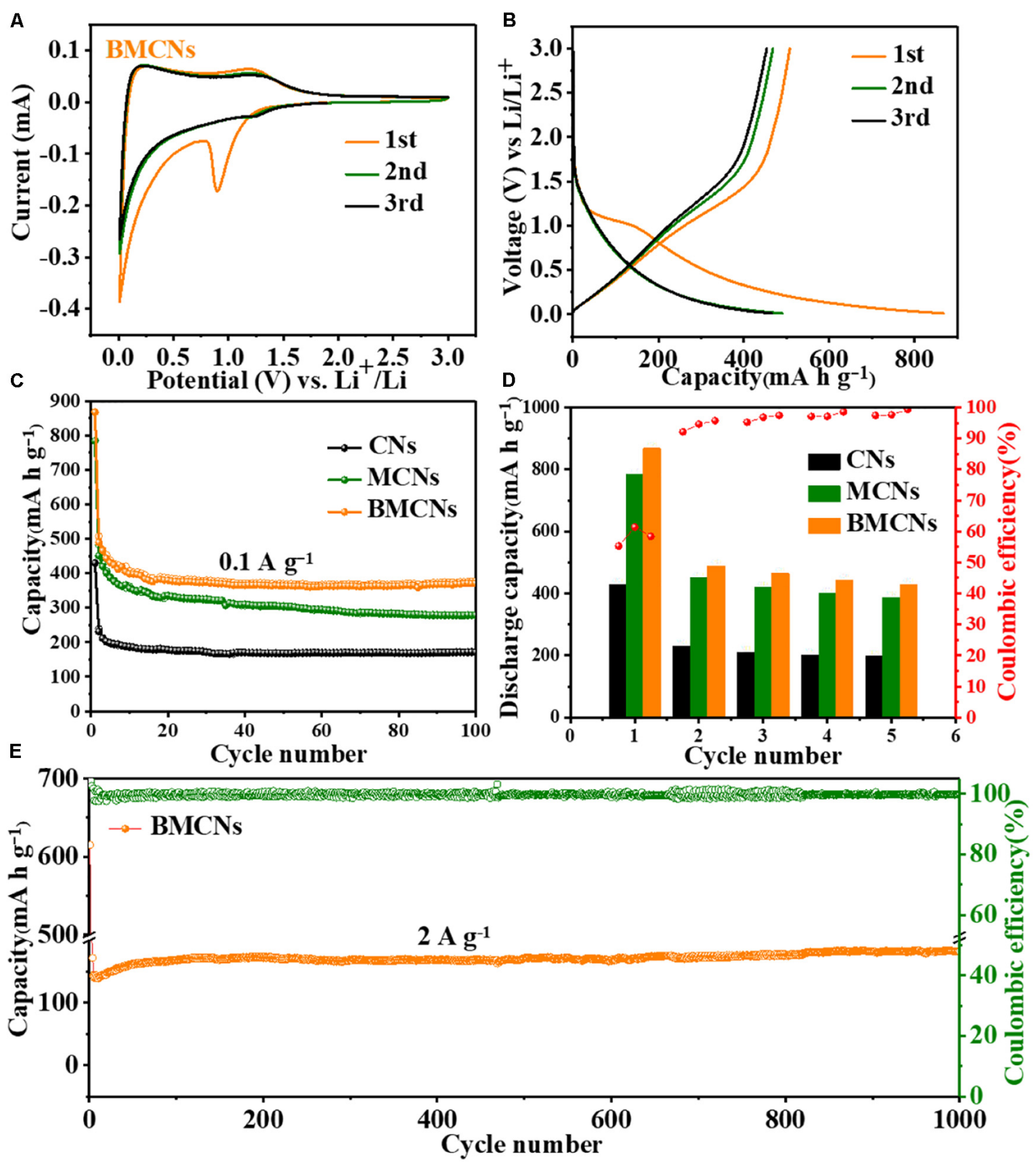

FIGURE 3 | (A) CV curves of the BMCN electrode at a voltage range of 0.01-3.0 V. (B) Charge/discharge curves of the BMCN electrode at a current density of $0.1 \mathrm{~A} \mathrm{~g}^{-1}$. (C) Cycling performance test of $\mathrm{CN}, \mathrm{MCN}$, and BMCN electrodes at the current density of $0.1 \mathrm{~A} \mathrm{~g}^{-1}$. (D) Discharge capacity and Coulombic efficiency of the first five cycles of $\mathrm{CN}, \mathrm{MCN}$, and BMCN electrodes at the current density of $0.1 \mathrm{~A} \mathrm{~g}^{-1}$. (E) Long-cycling performance and Coulombic efficiency of the BMCN electrodes at the high current density of $2 \mathrm{~A} \mathrm{~g}^{-1}$.

capacities. In addition, the Coulombic efficiency of the BMCN electrode is maintained at above $96 \%$ from the second cycle, which are superior to CNs and MCNs. The rate performance of $\mathrm{CNs}$, MCNs, and BMCNs at various current densities ranging from 0.1 to $5 \mathrm{~A} \mathrm{~g} \mathrm{~g}^{-1}$ is presented in Supplementary Figure $\mathbf{S 8}$. The BMCN electrode delivers reversible charge capacities of 448 , $381,339,285,243$, and $184 \mathrm{~mA} \mathrm{~h} \mathrm{~g}^{-1}$ at current densities of
$0.1,0.2,0.5,1,2$, and $5 \mathrm{~A} \mathrm{~g}^{-1}$, respectively, which are superior to CNs and MCNs. When the current density returns from 5 to $0.1 \mathrm{~A} \mathrm{~g}^{-1}$, a high charge capacity of $425 \mathrm{~mA} \mathrm{~h} \mathrm{~g}^{-1}$ can be recovered. To better demonstrate the good lithium ion storage performance of BMCNs at high current densities, the long cycling performance was further investigated at $2 \mathrm{~A} \mathrm{~g}^{-1}$ (Figure 3E), the BMCN electrode which delivered a reversible capacity of 

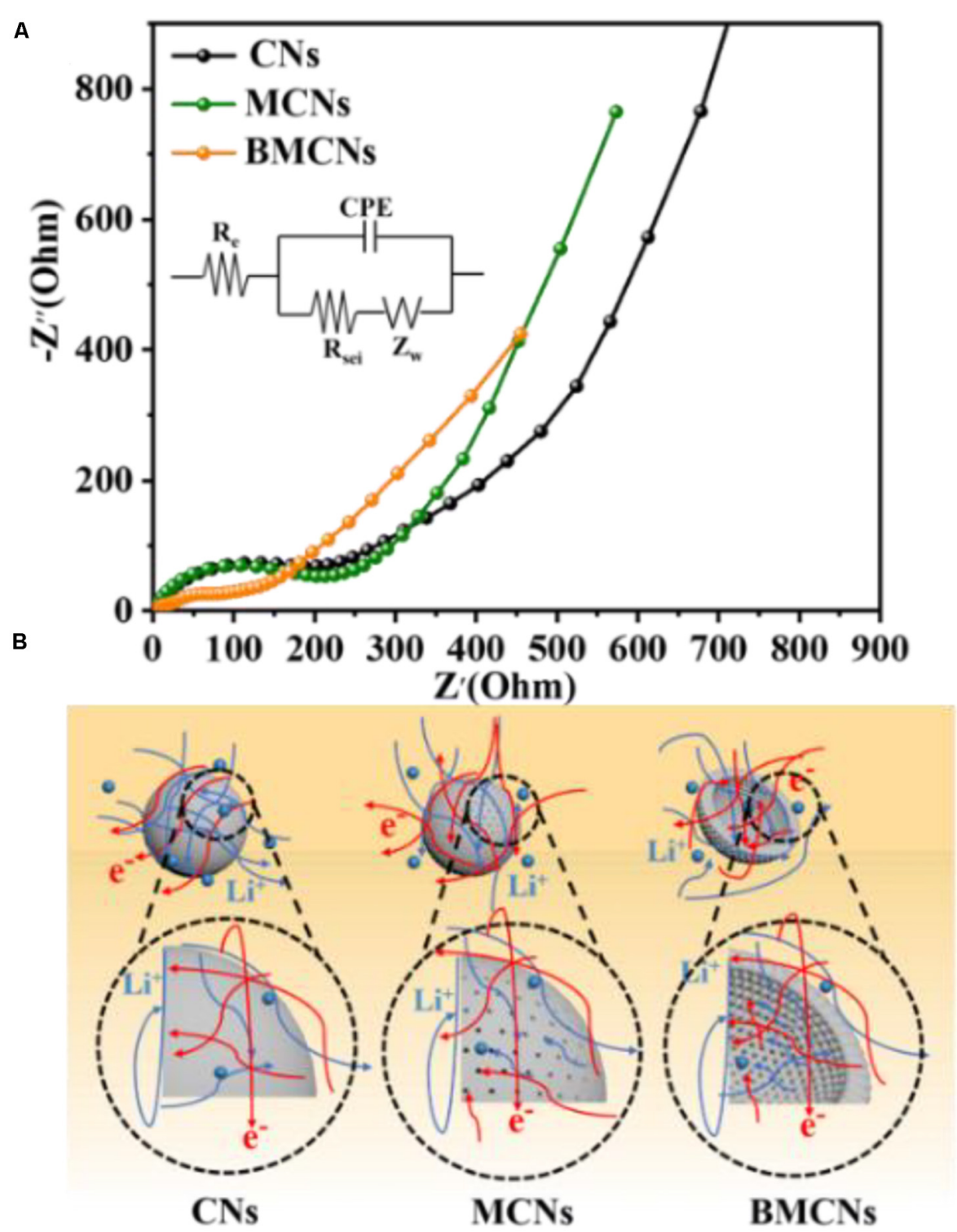

FIGURE 4 | (A) Electrochemical impedance spectra and equivalent circuit (inset) of the CN, MCN, and BMCN electrodes obtained after 50 cycles at 2 A g ${ }^{-1}$. (B) Schematic illustration for the lithium-ion and electron transport in the CN, MCN, and BMCN electrodes.

$300 \mathrm{~mA} \mathrm{~h} \mathrm{~g}^{-1}$ is retained, and the Coulombic efficiency closes to $100 \%$ after 1000 cycles, indicating outstanding cycling stability over a long cycle life.

The electrochemical impedance spectroscopy (EIS) measurements of three samples were conducted after the 50th cycles. All Nyquist plots are composed of a semicircle at the medium frequencies and an inclined line in the low-frequency region. The equivalent circuit is fitted using the ZSimpWin software. In general, the semicircle at the medium frequencies can be assigned to the interfacial transfer impedance of electrons $\left(R_{\mathrm{CT}}\right)$, and the slope spike denotes the diffusion ability of $\mathrm{Li}$ ions in the electrodes. $R_{\mathrm{e}}$ represents the electrolyte resistance. The CPE (constant phase element) denotes the capacitance of the SEI films and the double-layer capacitance, and $Z_{\mathrm{W}}$ is in relation with the Warburg impedance (Hao et al., 2018; Wu et al., 2019; Zhang et al., 2019). It can be observed that the semicircle of the BMCN electrode (Figure 4A) is smaller than other samples; this proves that the ion transport within the BMCN electrode is further ameliorated. The outstanding electrochemical performance of the BMCN electrode could be explained by the bowl-like mesoporous nanostructure (Figure 4B). Lithium ions can be stored reversibly between graphene-like planes. Comparing the electrochemical performance test results of CNs and MCNs indicates that the existence of mesoporous channels can provide convenient channels for lithium ions and electrons and accelerate the penetration of electrolytes, which can improve the electrochemical performance of electrodes. Differences in electrochemical performance between MCNs 
and BMCNs are mainly due to the unique advantages of the bowl-like morphology. In terms of geometry, this special bowl-like morphology has a shorter transmission distance than nanospheres, which can shorten the lithium ion and electron transmission paths.

In a nutshell, we successfully synthesized polydopaminederived carbon materials with different porosities or morphologies using three different methods. BMCNs were synthesized by an emulsion-induced self-assembly strategy, and MCNs were prepared according to a general nano-emulsion assembly method. CNs were synthesized by self-polymerization. Importantly, the effects of porosity and morphology on their electrochemical performance under the same conditions were studied in detail. Electrochemical test results show that the mesoporous structure can shorten the diffusion path of $\mathrm{Li}^{+}$and electrons in the electrode and accelerate the diffusion rate of the electrolyte. In addition, the special bowl-like morphology has a shorter transmission distance than nanospheres, which can shorten the lithium ion and electron transmission paths.

\section{DATA AVAILABILITY STATEMENT}

The raw data supporting the conclusions of this article will be made available by the authors, without undue reservation.

\section{REFERENCES}

Ai, K., Liu, Y., Ruan, C., Lu, L., and Lu, G. (2013). Sp² C-dominant N-doped carbon sub-micrometer spheres with a tunable size: a versatile platform for highly efficient oxygen-reduction catalysts. Adv. Mater. 25, 998-1003. doi: 10. 1002/adma.201203923

Boyjoo, Y., Shi, H., Olsson, E., Cai, Q., Wu, Z.-S., Liu, J., et al. (2020). Molecularlevel design of pyrrhotite electrocatalyst decorated hierarchical porous carbon spheres as nanoreactors for lithium-sulfur batteries. Adv. Energy Mater. 10:2000651. doi: 10.1002/aenm.202000651

Chen, J., Mao, Z., Zhang, L., Tang, Y., Wang, D., Bie, L., et al. (2018). Direct production of nitrogen-doped porous carbon from urea via magnesiothermic reduction. Carbon 130, 41-47. doi: 10.1016/j.carbon.2017. 12.125

Chen, Y.-Y., Zhang, Y., Jiang, W.-J., Zhang, X., Dai, Z., Wan, L.-J., et al. (2016). Pomegranate-like N,P-Doped $\mathrm{Mo}_{2} \mathrm{C} @ \mathrm{C}$ nanospheres as highly active electrocatalysts for alkaline hydrogen evolution. ACS Nano 10, 8851-8860. doi: 10.1021/acsnano.6b04725

Chu, H., Shao, C., Qiu, S., Zou, Y., Xiang, C., Xu, F., et al. (2018). Nitrogen-rich sandwich-like carbon nanosheets as anodes with superior lithium storage properties. Inorg. Chem.Front. 5, 225-232. doi: 10.1039/C7QI0 0567A

Cui, Y., Zhou, X., Guo, W., Liu, Y., Li, T., Fu, Y., et al. (2019). Selenium nanocomposite cathode with long cycle life for rechargeable lithium-selenium batteries. Batt. Super. 2, 784-791. doi: 10.1002/batt.201900050

Dai, X.-H., Fan, H.-X., Zhang, J.-J., and Yuan, S.-J. (2019). Sewage sludge-derived porous hollow carbon nanospheres as high-performance anode material for lithium ion batteries. Electrochim. Acta 319, 277-285. doi: 10.1016/j.electacta. 2019.07.006

Gayathri, S., Arunkumar, P., Kim, E. J., Kim, S., Kang, I., and Han, J. H. (2019). Mesoporous nitrogen-doped carbon@graphene nanosheets as ultrastable anode for lithium-ion batteries - Melamine as surface modifier than nitrogen source. Electrochim. Acta 318, 290-301. doi: 10.1016/j.electacta.2019. 06.054

Hao, J., Wang, Y., Chi, C., Wang, J., Guo, Q., Yang, Y., et al. (2018). Enhanced storage capability by biomass-derived porous carbon for lithium-ion and

\section{AUTHOR CONTRIBUTIONS}

XQ completed the experiments and test. FZ prepared the draft manuscript. YZ discussed the structures. KL and WL polished the manuscript. JY designed this manuscript. All authors contributed to the article and approved the submitted version.

\section{FUNDING}

This work was financially supported by the Fok Ying-Tong Education Foundation of China (No. 171041), the Program for Professor of Special Appointment (Eastern Scholar) at Shanghai Institutions of Higher Learning, the State Key Laboratory for Modification of Chemical Fibers and Polymer Materials, Donghua University, and DHU Distinguished Young Professor Program and Fundamental Research Funds for the Central Universities.

\section{SUPPLEMENTARY MATERIAL}

The Supplementary Material for this article can be found online at: https://www.frontiersin.org/articles/10.3389/fenrg. 2020.00140/full\#supplementary-material

sodium-ion battery anodes. Sustain. Energy Fuels 2, 2358-2365. doi: 10.1039/ C8SE00353J

Hong, Y.-S., Zhao, C.-Z., Xiao, Y., Xu, R., Xu, J.-J., Huang, J.-Q., et al. (2019). Safe lithium-metal anodes for $\mathrm{Li}^{-} \mathrm{O}_{2}$ batteries: from fundamental chemistry to advanced characterization and effective protection. Batt. Super. 2, 638-658. doi: 10.1002/batt.201900031

Hu, X., Sun, X., Yoo, S. J., Evanko, B., Fan, F., Cai, S., et al. (2019). Nitrogenrich hierarchically porous carbon as a high-rate anode material with ultra-stable cyclability and high capacity for capacitive sodium-ion batteries. Nano Energy 56, 828-839. doi: 10.1016/j.nanoen.2018.11.081

Jian, Z., Li, H., Cao, R., Zhou, H., Xu, H., Zhao, G., et al. (2019). Polydopaminecoated hierarchical tower-shaped carbon for high-performance lithium-sulfur batteries. Electrochim. Acta 319, 359-365. doi: 10.1016/j.electacta.2019.06.145

Jiang, H., Ren, D., Wang, H., Hu, Y., Guo, S., Yuan, H., et al. (2015). 2D monolayer $\mathrm{MoS}_{2}$-carbon interoverlapped superstructure: engineering ideal atomic interface for lithium ion storage. Adv. Mater. 27, 3687-3695. doi: 10. 1002/adma.201501059

Lang, J., Li, J., Zhang, F., Ding, X., Zapien, J. A., and Tang, Y. (2019). Sodium-ion hybrid battery combining an anion-intercalation cathode with an adsorptiontype anode for enhanced rate and cycling performance. Batt. Super. 2, 440-447. doi: 10.1002/batt.201800138

Li, L., Zhang, W., Wang, X., Zhang, S., Liu, Y., Li, M., et al. (2019). Hollowcarbon-templated few-layered $\mathrm{V}_{5} \mathrm{~S}_{8}$ nanosheets enabling ultrafast potassium storage and long-term cycling. ACS Nano 13, 7939-7948. doi: 10.1021/acsnano. $9 \mathrm{~b} 02384$

Liang, J., Hu, H., Park, H., Xiao, C., Ding, S., Paik, U., et al. (2015). Construction of hybrid bowl-like structures by anchoring $\mathrm{NiO}$ nanosheets on flat carbon hollow particles with enhanced lithium storage properties. Energy Envion. Sci. 8, 1707-1711. doi: 10.1039/C5EE01125F

Liu, C., Xiao, N., Wang, Y., Zhou, Y., Wang, G., Li, H., et al. (2018). Electrospun nitrogen-doped carbon nanofibers with tuned microstructure and enhanced lithium storage properties. Carbon 139, 716-724. doi: 10.1016/j.carbon.2018. 07.019

Liu, H., Liu, X., Li, W., Guo, X., Wang, Y., Wang, G., et al. (2017). Porous carbon composites for next generation rechargeable lithium batteries. Adv. Energy Mater. 7:1700283. doi: 10.1002/aenm.201700283 
Liu, J., Wickramaratne, N. P., Qiao, S. Z., and Jaroniec, M. (2015). Molecular-based design and emerging applications of nanoporous carbon spheres. Nat. Mater. 14, 763-774. doi: 10.1038/nmat4317

Liu, J., Xu, X., Hu, R., Yang, L., and Zhu, M. (2016). Uniform hierarchical $\mathrm{Fe}_{3} \mathrm{O}_{4} @$ polypyrrole nanocages for superior lithium ion battery anodes. $A d v$. Energy Mater. 6:1600256. doi: 10.1002/aenm.201600256

Liu, N., Wu, H., McDowell, M. T., Yao, Y., Wang, C., and Cui, Y. (2012). A yolkshell design for stabilized and scalable Li-ion battery alloy anodes. Nano Lett. 12, 3315-3321. doi: 10.1021/nl3014814

Liu, S., Mao, J., Zhang, Q., Wang, Z., Pang, W. K., Zhang, L., et al. (2020). An intrinsically non-flammable electrolyte for high-performance potassium batteries. Angew. Chem., Int. Ed. 59, 3638-3644. doi: 10.1002/anie.201913174

Mao, J., Zhou, T., Zheng, Y., Gao, H., Liu, H. K., and Guo, Z. (2018). Twodimensional nanostructures for sodium-ion battery anodes. J. Mater. Chem. A 6, 3284-3303. doi: 10.1039/C7TA10500B

Nan, J., Guo, X., Xiao, J., Li, X., Chen, W., Wu, W., et al. (2019). Nanoengineering of 2D MXene-based materials for energy storage applications. Small 1902085. doi: 10.1002/smll.201902085 [Epub ahead of print].

Ould Ely, T., Kamzabek, D., and Chakraborty, D. (2019). Batteries safety: recent progress and current challenges. Front. Energy Res. 7:71. doi: 10.3389/fenrg. 2019.00071

Peng, L., Hung, C.-T., Wang, S., Zhang, X., Zhu, X., Zhao, Z., et al. (2019). Versatile nanoemulsion assembly approach to synthesize functional mesoporous carbon nanospheres with tunable pore sizes and architectures. J. Am. Chem. Soc. 141, 7073-7080. doi: 10.1021/jacs.9b02091

Qian, X., Zhu, G., Wang, K., Zhang, F., Liang, K., Luo, W., et al. (2020). Bowl-like mesoporous polymer-induced interface growth of molybdenum disulfide for stable lithium storage. Chem. Eng. J. 381:122651. doi: 10.1016/j.cej.2019.122651

Qie, L., Chen, W.-M., Wang, Z.-H., Shao, Q.-G., Li, X., Yuan, L.-X., et al. (2012). Nitrogen-doped porous carbon nanofiber webs as anodes for lithium ion batteries with a superhigh capacity and rate capability. Adv. Mater. 24, 2047-2050. doi: 10.1002/adma.201104634

Rao, X., Lou, Y., Chen, J., Lu, H., Cheng, B., Wang, W., et al. (2020). Polyacrylonitrile hard carbon as anode of high rate capability for lithium ion batteries. Front. Energy Res. 8:3. doi: 10.3389/fenrg.2020.00003

Shen, S., Xia, X., Zhong, Y., Deng, S., Xie, D., Liu, B., et al. (2019). Implanting niobium carbide into trichoderma spore carbon: a new advanced host for sulfur cathodes. Adv. Mater. 31:1900009. doi: 10.1002/adma.201900009

Shi, M., Nie, P., Fan, Z., Fu, R., Fang, S., Dou, H., et al. (2020). Tubular graphene nano-scroll coated silicon for high rate performance lithium-ion battery. Front. Energy Res. 8:2. doi: 10.3389/fenrg.2020.00002

Sun, G., Zhang, F., Xie, Q., Luo, W., and Yang, J. (2019). Regulating ambient pressure approach to graphitic carbon nitride towards dispersive layers and rich pyridinic nitrogen. Chin. Chem. Lett. doi: 10.1016/j.cclet.2019.10.018 [Epub ahead of print].

Tarascon, J. M., and Armand, M. (2001). Issues and challenges facing rechargeable lithium batteries. Nature 414, 359-367. doi: 10.1038/35104644

Tian, H., Liang, J., and Liu, J. (2019). Nanoengineering carbon spheres as nanoreactors for sustainable energy applications. Adv. Mater. 31:1903886. doi: 10.1002/adma.201903886

Wang, L., Lu, B., Wang, S., Cheng, W., Zhao, Y., Zhang, J., et al. (2019). Ultrahigh performance of $\mathrm{Li} / \mathrm{Na}$ ion batteries using N/O dual dopant porous hollow carbon nanocapsules as an anode. J. Mater. Chem. A 7, 11117-11126. doi: 10.1039/C8TA12540F
Wang, N., Hou, D., Li, Q., Zhang, P., Wei, H., and Mai, Y. (2019). Two-dimensional interface engineering of mesoporous polydopamine on graphene for novel organic cathodes. ACS Appl. Energy Mater. 2, 5816-5823. doi: 10.1021/acsaem. 9 b00946

Wang, Z., Qiang, H., Zhu, Z., Liu, J., Chen, C., and Zhang, D. (2018). Facile synthesis of nitrogen-doped mesoporous hollow carbon nanospheres for highperformance supercapacitors. ChemElectroChem 5, 2242-2249. doi: 10.1002/ celc. 201800597

Wu, J., Cao, Y., Zhao, H., Mao, J., and Guo, Z. (2019). The critical role of carbon in marrying silicon and graphite anodes for high-energy lithium-ion batteries. Carbon Energy 1, 57-76. doi: 10.1002/cey2.2

Xu, B., Qi, S., Jin, M., Cai, X., Lai, L., Sun, Z., et al. (2019). 2020 roadmap on twodimensional materials for energy storage and conversion. Chin. Chem. Lett. 30, 2053-2064. doi: 10.1016/j.cclet.2019.10.028

Yin, D., Zhao, H., Li, N., Si, R., Sun, X., Li, X., et al. (2019). Enhancing the rate capability of niobium oxide electrode through rare-earth doping engineering. Batt. Super. 2, 924-928. doi: 10.1002/batt.201900069

Zhang, F., Zhu, G., Wang, K., Qian, X., Zhao, Y., Luo, W., et al. (2019) Boosting the initial coulombic efficiency in silicon anodes through interfacial incorporation of metal nanocrystals. J. Mater. Chem. A 7, 17426-17434. doi: 10.1039/C9TA05340A

Zhang, L., Sun, D., Kang, J., Feng, J., Bechtel, H. A., Wang, L.-W., et al. (2018). Electrochemical reaction mechanism of the $\mathrm{MoS}_{2}$ electrode in a lithium-ion cell revealed by in situ and operando X-ray absorption spectroscopy. Nano Lett. 18, 1466-1475. doi: 10.1021/acs.nanolett.7b05246

Zhao, Q., Meng, Y., Li, J., and Xiao, D. (2019). Sulfur and nitrogen dual-doped porous carbon nanosheet anode for sodium ion storage with a self-template and self-porogen method. Appl. Surface Sci. 481, 473-483. doi: 10.1016/j.apsusc. 2019.03.143

Zheng, C., Hu, X., Sun, X., Yoo, S. J., and Li, X. (2019). Large-scale synthesis of nitrogen-rich hierarchically porous carbon as anode for lithium-ion batteries with high capacity and rate capability. Electrochim. Acta 306, 339-349. doi: 10.1016/j.electacta.2019.03.137

Zheng, F., Yang, Y., and Chen, Q. (2014). High lithium anodic performance of highly nitrogen-doped porous carbon prepared from a metal-organic framework. Nat. Communi. 5:5261. doi: 10.1038/ncomms6261

Zhu, G., Zhang, F., Li, X., Luo, W., Li, L., Zhang, H., et al. (2019). Engineering the distribution of carbon in silicon oxide nanospheres at the atomic level for highly stable anodes. Angew. Chem. Int. Ed. 58, 6669-6673. doi: 10.1002/anie. 201902083

Zou, G., Hou, H., Foster, C. W., Banks, C. E., Guo, T., Jiang, Y., et al. (2018). Advanced hierarchical vesicular carbon co-doped with S, P, N for high-rate sodium storage. Adv. Sci. 5:1800241. doi: 10.1002/advs.201800241

Conflict of Interest: The authors declare that the research was conducted in the absence of any commercial or financial relationships that could be construed as a potential conflict of interest.

Copyright (c) 2020 Qian, Zhang, Zhao, Liang, Luo and Yang. This is an open-access article distributed under the terms of the Creative Commons Attribution License (CC BY). The use, distribution or reproduction in other forums is permitted, provided the original author(s) and the copyright owner(s) are credited and that the original publication in this journal is cited, in accordance with accepted academic practice. No use, distribution or reproduction is permitted which does not comply with these terms. 